\title{
Brain transcriptome profiling reveals involvement of divergent pathways in C9orf72-associated and sporadic ALS
}

The pathogenetic mechanisms involved in amyotrophic lateral sclerosis (ALS) caused by C9orf72 repeat expansions (c9 ALS) could differ from those of sporadic ALS, according to a new transcriptome profiling study. The findings underscore the aetiological diversity of ALS, and the need for subtype-specific treatments.

\section{1 ...though the clinical presentations are similar, the underlying contributing factors could be different... 77}

Although the majority of ALS cases are sporadic, about $10 \%$ of patients with ALS have a familial form of the disease. C9orf72 repeat expansions are the most common inherited mutations accounting for familiar ALS, and are also present in $5-6 \%$ of patients with sporadic ALS. The repeat expansions are thought to disrupt RNA processing, but the pathogenetic mechanisms of $\mathrm{c} 9$ and other ALS subtypes are incompletely understood.

Leonard Petrucelli, Hu Li and colleagues used next-generation RNA sequencing to analyze postmortem samples of cerebellum and frontal cortex of eight patients with c9 ALS, 10 patients with sporadic ALS without C9orf72 mutations, and eight controls without neurological disease. The researchers observed extensive misregulation of alternative splicing, polyadenylation and gene expression in both c9 and sporadic ALS.

Interestingly, the changes in the transcriptome were particularly pronounced in the cerebellum. Cerebellar atrophy has been reported in patients with ALS, but the contribution of cerebellar pathology to ALS is poorly understood.

Some of the transcriptomic alterations were shared between the two ALS groups, but many of the affected pathways were group-specific. C9 ALS was characterized by changes in transcripts involved in the unfolded protein response and nuclear transport, whereas in sporadic ALS, synaptic transmission and organization of the cytoskeleton were primarily affected.

"The findings indicate that though the clinical presentations of $\mathrm{c} 9$ ALS and sporadic ALS are similar, the underlying contributing factors could be different," summarizes Petrucelli.

The results might provide important clues to the pathogenetic mechanisms involved in ALS. Next, the researchers plan to apply their findings to the identification of potential disease biomarkers, which could help monitor disease progression and response to potential therapies.

\section{Hemi Malkki}

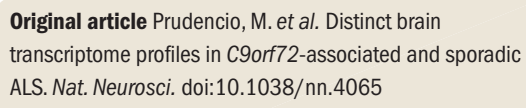

Further reading Paez-Colasante, X. et al. Amyotrophic lateral sclerosis: mechanisms and therapeutics in the epigenomic era. Nat. Rev. Neurol. 11, 266-279 (2015) 